\title{
L'altermondialisme et la recherche sur les mouvements sociaux. Quelques réflexions
}

Alterglobalism and the research on social movements. Some reflections

\section{Donatella Della Porta}

Traducteur : Ciro Reni et Deniz Günce Demirhisar

\section{OpenEdition \\ Journals}

Édition électronique

URL : http://journals.openedition.org/conflits/12293

DOI : $10.4000 /$ conflits. 12293

ISSN : $1777-5345$

Éditeur :

CCLS - Centre d'études sur les conflits lilberté et sécurité, L'Harmattan

\section{Édition imprimée}

Date de publication : 20 juillet 2008

Pagination : 13-31

ISBN : 978-2-296-05909-2

ISSN : $1157-996 X$

Référence électronique

Donatella Della Porta, «L'altermondialisme et la recherche sur les mouvements sociaux. Quelques réflexions », Cultures \& Conflits [En ligne], 70 | été 2008, mis en ligne le 10 septembre 2008, consulté le 30 mars 2021. URL : http://journals.openedition.org/conflits/12293 ; DOI : https://doi.org/10.4000/ conflits. 12293 


\section{L'altermondialisme et la recherche sur les mouvements sociaux. Quelques réflexions}

\section{Donatella DELLA PORTA}

Donatella della Porta est professeur de sociologie à l'Institut universitaire européen de Florence. Elle a récemment publié : Globalization from Below, The University of Minnesota Press, 2006 ; The Policing of Transnational Protest, Ashgate; The Global Justice Movement, Paradigm.

T e 30 novembre 1999, à Seattle, ville américaine devenue le symbole de la LNew Economy, environ 50000 personnes protestent contre la 3e conférence de l'Organisation mondiale du commerce (OMC) réunie pour lancer le Millenium Round, un nouveau cycle de négociations visant à poursuivre la libéralisation du marché, en particulier celles des investissements et des services publics ${ }^{1}$. Les manifestations de Seattle avaient été lancées en 1999 à Genève par une coordination d'organisations de provenances diverses, qui s'étaient déjà mobilisées avec succès pour empêcher la signature d'un traité international, le Multilateral Agreement on Investment (MAI), accusé de réduire, au nom du libre-échange, la capacité des Etats à intervenir sur les enjeux sociaux et environnementaux. Les manifestations contre le Millenium Round - précédées d'assemblées et d'initiatives de diffusion d'informations dans le monde entier ont été organisées par 1387 ONG, syndicats, écologistes et églises de différentes confessions. Parmi les slogans des manifestations, on trouve ceux-ci : «The world is not for sale "; "No globalization without participation"; "We are citizens, not only consumers "; «WTO = Capitalism without conscience »; «Trade: clean, green and fair» 2 .

Les rassemblements de Seattle ont été définis comme un moment charnière, mais aussi comme le point culminant d'un processus d'intégration de

1. Traduit de l'italien par Ciro Reni et Deniz Günce Demirhisar.

2. NdT : «Le monde n'est pas à vendre ", "Pas de mondialisation sans participation », "Nous sommes des citoyens, pas simplement des consommateurs ", "OMC = capitalisme sans conscience », «Le commerce : propre, vert et juste ». 
groupes et d'organisations actifs dans plusieurs parties du monde: des ouvriers des pays riches ou pauvres et des paysans; des consommateurs et des écologistes; des églises et des féministes; des pacifistes et des associations de défense des droits de l'Homme. Ceci peut constituer le point de départ de ce que nous allons ici développer : une réflexion sur les caractéristiques du mouvement altermondialiste du Nord et, par-là même, sur les défis que présente la recherche sur l'altermondialisme dans la partie sud de la planète.

\section{Un mouvement social spontané}

Rappelons tout d'abord qu'on a observé bien avant Seattle des mobilisations de groupes hétérogènes au départ peu connectés, qui dirigeaient leur action tout particulièrement contre certaines organisations internationales.

L’OMC a été la cible des contestations des écologistes, qui la jugeaient responsable de plusieurs violations des accords de libéralisation des marchés qu'elle aurait elle-même établis. Ces accusations étaient fondées sur plusieurs éléments : le non-respect de la part des Etats-Unis de l'interdiction de l'importation de crevettes pêchées par des filets n'ayant pas de «Dispositif d'exclusion des tortues », un système qui permet aux tortues de mer de se libérer; le non-respect par le Japon et l'Europe de l'interdiction d'importer des produits traités par pesticides pour l'un, et de la viande d'animaux nourris aux hormones pour l'autre ; la pratique frauduleuse au Canada, qui consiste à permettre le mélange de méthanol et d'essence. Par ailleurs, les paysans indiens avaient manifesté dès 1990 contre les brevets sur les semences et les organismes génétiquement modifiés, qui étaient soutenus par l'OMC.

Les organisations de défense des consommateurs se sont mobilisées contre les accords supranationaux (l'Accord de libre échange nord-américain, ALENA ; l'Accord général sur les tarifs douaniers et le commerce, le GATT; l'OMC) accusés de réduire, au nom du libre-échange, les standards de protection des citoyens. Lors des conférences de l'ONU portant sur les droits des femmes, les organisations féministes du Nord et du Sud de la planète se sont rencontrées. Les ONG militant en faveur du développement ont exercé des pressions afin d'obtenir une augmentation des aides allouées au Tiers-monde, allant jusqu'à revendiquer "la réparation des dettes historiques, sociales et écologiques » imposées par le Nord au Sud (comme la réparation du colonialisme). Impliquant surtout, mais non exclusivement, les groupes religieux, la campagne Jubileum 2000 a demandé l'annulation des dettes des pays les plus pauvres. Aux Etats-Unis en particulier, avec l'appui notamment des communautés afro-américaines, la dénonciation des conditions extrêmes d'exploitation, notamment celle des femmes et des enfants, dans des sweatshops du Sud, a été très forte, tandis que les syndicats du Nord et du Sud ont demandé l'introduction d'instruments de promotion et de défense des droits des travailleurs dans les traités internationaux. De multiples organisations pacifistes ainsi 
que des organisations pour la défense des droits de l'Homme, pour la liberté de circulation des migrants, ou encore contre les mines antipersonnelles se sont réunies à l'occasion de ces campagnes.

Le thème commun de toutes ces manifestations est la critique d'une sorte de dégénérescence de l'économie de marché, advenue suite à l'hégémonie acquise par les doctrines économiques néolibérales à partir des années 1980. Au nom du libre-échange, on aurait notamment renoncé à la défense des droits sociaux qui sont devenus parties intégrantes - du moins dans les pays du Nord - de la définition même des droits de la citoyenneté. En outre, alors que beaucoup d'économistes énuméraient les avantages de l'élimination des barrières protectionnistes pour le Sud, des « contre-experts », mobilisés dans l'opposition, ont rétorqué qu'il existait également des effets d'ensemble négatifs dans les pays en voie de développement. Par conséquent, les protestations se sont retournées contre ce qu'une experte en relations internationales, Susan Strange, a décrit comme un "corporation empire ", composé d'une bureaucratie impériale, à la tête de laquelle se trouvent le département du trésor américain et les multinationales, qui ont le contrôle sur la direction des organisations financières internationales :

«l'autorité de cet Empire non territorial est exercée directement sur les personnes et non sur les territoires. Elle est exercée sur les banquiers et sur les dirigeants des corporations, sur les épargnants et les investisseurs, sur les journalistes et les professeurs. Elle est aussi naturellement exercée sur les plus hautes autorités des gouvernements alliés et associés ${ }^{3}$.»

Ces diverses campagnes ont en commun le fait de considérer la libéralisation des marchés non pas comme un effet « naturel » du développement technologique, mais plutôt comme une stratégie avantageuse pour les entreprises multinationales, adoptée et défendue par les institutions financières internationales (la Banque mondiale, le Fond monétaire international et l'Organisation mondiale du commerce) et par les gouvernements des pays les plus puissants (notamment à travers le G7 et le G8).

De ce point de vue, les organisations prenant part aux manifestations ont souvent réfuté la dénomination de «noglobal », en lui préférant celle d' "altermondialiste » : en demandant une mondialisation différente, ces organisations ne s'opposent pas à l'intensification des échanges culturels et au développement des structures de gouvernance supranationales. Elles contestent plutôt les choix spécifiques, néolibéraux, de ces institutions, qui se substituent à ceux des gouvernements nationaux. C'est surtout après Seattle que la critique des

3. Strange S., "Toward a theory of transnational empire”, in Czempiel E.O., J.N. Rosenau (eds.), Global Change and Theoretical Challenges, Lexington, Lexington Books, p. 170. 
formes néolibérales de la mondialisation et la demande d'une «autre mondialisation » entrent dans le débat public - comme on peut le lire dans un éditorial de l'hebdomadaire américain Nerwsweek:

«Jusqu'à aujourd'hui, il était facile d'affirmer que quiconque opposé au libre-échange était par définition un protectionniste, content de se cacher derrière les cloisons de l'Etat-nation. Cette simple équation ne tient plus ; l'une des plus importantes leçons de Seattle, c'est qu'il y a désormais deux visions de la mondialisation qui sont proposées : l'une guidée par le commerce, l'autre par l'activisme social ${ }^{4}$. »

Ainsi, après Seattle, les références à un mouvement mondial sont de plus en plus fréquentes. Certes, la majorité des manifestants présents était nord-américaine (d'après certaines estimations, 20000 à 25000 manifestants venaient de l'Etat de Washington même; 15000 à 20000 d'autres Etats américains ; 3000 à 5000 du Canada). Mais le caractère international des manifestations est nettement confirmé par les initiatives organisées en parallèle dans d'autres centresvilles au Nord et au Sud, dans le cadre de ce qui sera appelé la Journée d'action globale (Global Day Action). A près Seattle, les protestations concernant la mondialisation se sont poursuivies dans des dizaines de pays, en acquérant toujours plus de visibilité. A partir de Seattle, chaque sommet international relativement important a été accompagné de manifestations qui, souvent, ont été plus relayées par les journaux que le programme officiel de ces sommets. Parmi les nombreuses manifestations organisées en 2000 , on peut particulièrement noter celles contre le Forum économique mondial de Davos en janvier; contre la Banque mondiale et le FMI en avril ; au sommet de l'ONU sur la pauvreté à Genève en juin ; à la réunion du FMI et de la Banque mondiale à Prague en septembre; au sommet de l'UE à Nice en décembre. L'année suivante, les contestations n'ont guère perdu de leur ampleur. En effet, des manifestations se sont déroulées de nouveau en février, à l'occasion du Forum économique mondial de Davos; en avril, à Québec, contre le traité du FTA (Free Trade of the Americas); à Göteborg au sommet de l'UE en juin, puis en juillet à celui du G7 à Gênes. On peut dire qu'à partir de Seattle, le mouvement contestataire a, du moins, réussi à faire sortir les négociations internationales de l'ombre des accords discrets entre diplomates et technocrates, en leur donnant une visibilité médiatique : "jamais auparavant, les débuts des négociations multilatérales sur le commerce n'avaient été au coeur de la sphère publique internationale 5 ».

A côté des controverses, des rencontres sur une autre «mondialisation » possible se tiennent lors des forums sociaux mondiaux, avec une participation

4. Newsweek, 13 décembre 1999, p. 28. Toutes les citations de cet article ont été traduites par Ciro Reni et Deniz Günce Demirhisar.

5. Pfeil A., "Lehren aus Seattle », Blätter für dentsche und internationale Politik, vol.45, n`1, 2000 , p. 16. 
croissante : de 16400 inscrits à la première rencontre en janvier 2001, on est passé à 52000 en 2002, pour atteindre jusqu'à 150000 inscrits aux éditions les plus récentes ${ }^{6}$. A l'occasion des milliers de séminaires et d'assemblées, des propositions - plus ou moins réalistes et originales - sont élaborées pour une "mondialisation par le bas » et des politiques alternatives, dont certaines sont déjà en cours d'expérimentation, sont discutées. C'est surtout à partir de 2002 que l'expérience des forums sociaux comme lieux de rencontres et de débats s'étend au niveau local, ainsi qu'au niveau macro-régional. C'est à Florence plus particulièrement qu'a lieu, à l'automne de cette même année, le premier Forum social européen, avec trois jours de séminaires auxquels participent près de 60000 personnes. Quatre autres forums sociaux européens lui succèdent : à Paris en 2003, à Londres en 2004, à Athènes en 2006. Le cinquième est en cours de préparation pour septembre 2008, à Malmö. Le développement de cette protestation à vocation mondiale n'avait été prévu ni par les chercheurs ni par les commentateurs. Au début, bien des analyses sur la mondialisation étaient enclines à prévoir avec pessimisme la fin de ces rassemblements. On voyait dans les phénomènes liés à la mondialisation, et notamment les nouveaux rapports introduits dans le développement des actions collectives, la cause de ce déclin.

Les réflexions sur la « post-modernité » avaient surtout souligné les processus d'individualisation, qui auraient isolé l'individu de la communauté, accentuant l'égoïsme et, par conséquent, la difficulté d'agir ensemble. Par exemple, d'après l'analyse de Zygmunt Bauman, dans les sociétés contemporaines, la crise des réseaux traditionnels de sécurité produit une rupture de la solidarité, ce qui entraîne une multiplication des angoisses existentielles qui brident l'action collective.

«Dans toute société, la solidarité, ou plutôt les denses réseaux de solidarité (qu'ils soient grands ou petits, juxtaposés ou croisés) ont servi à la protection et à la garantie de certitude (bien qu'imparfaite), en produisant la confiance, l'assurance en soi et le courage indispensables à l'exercice de la liberté et à l'expérimentation. La principale victime de la théorie et de la pratique néolibérales a été cette solidarité même ${ }^{7}$. »

Il en ressort la conviction selon laquelle «les individus ne peuvent pas faire grand-chose, personnellement ou collectivement, pour contrer, ou du moins vaincre, les menaces à la sécurité de leurs conditions sociales ou à la certitude de leurs perspectives d'avenir ». Les tentatives pour localiser les menaces aboutissent à "la résignation ou la dispersion, à l'attitude du genre: "Non, il n'y a rien que je puisse faire» 8 ». Après la chute du Mur de Berlin, dans un système international 
unipolaire, il n'y aurait pas eu de place pour de grandes utopies alternatives à celles du marché. La contestation se serait fragmentée dans toutes les campagnes à thématique unique et de court terme ${ }^{9}$. Pendant les années 1990, on a même parlé d'une crise des mouvements sociaux comme "une partie du paradigme plus large de crise globale de la politique et en particulier occidentale, qui implique la société civile et l'Etat suite à la fin de la Guerre froide 10 ».

La possibilité d'action collective aurait également été réduite par la délocalisation de la production dans les pays où les salaires sont plus bas et la protection des travailleurs est inférieure, ainsi que par la nécessité de maintenir la compétitivité, d'attirer des capitaux fluctuants à travers la flexibilisation du marché du travail. Si, à partir des années 1960, se sont succédés des travaux et des recherches proclamant la fin d'une conscience (et d'une lutte) de classe, la mondialisation même, avec la délocalisation de la production et le chômage qui s'ensuit, serait à l'origine d'un affaiblissement structurel de la force de travail, ainsi que d'une chute constante des taux de syndicalisation. La flexibilisation et les métiers « atypiques » apparaissent légitimés par une attente de succès individuel, temporairement soutenue par le dynamisme de certains secteurs de la nouvelle économie. Le mécontentement des chômeurs est toujours plus difficile à organiser dans le cadre d'actions de protestation collective. En général, les groupes socialement les plus faibles - les « victimes » de la mondialisation ont perdu la protection politique lorsque les partis et les gouvernements de centre-gauche ont accepté les politiques néolibérales de déréglementation et de réduction des dépenses sociales pour éviter la fuite des capitaux. De ce point de vue, la mondialisation économique aurait remis en cause non seulement le rôle de l'Etat national, que l'on considère de moins en moins capable de gouverner à l'intérieur de ses propres frontières, mais aussi, plus généralement, la capacité du politique à intervenir dans l'économie et à réguler les conflits sociaux. Dans cette image, l'espace du politique serait limité à sa capacité d'attirer les capitaux : le capitalisme mondial aurait rompu l'alliance historiquement consolidée entre le capitalisme, l'Etat-providence et la démocratie.

La capacité de mobilisation des mouvements sociaux, acteurs typiquement nationaux, serait en fin de compte diminuée face à une "internationalisation significative de l'autorité politique en même temps que la mondialisation de cette même autorité politique $11 »-$ avec une augmentation du nombre d'organisations intergouvernementales, qui sont passées de 37 en 1909 (année du premier recensement) à 1592 en $1997^{12}$. Avec l'accroissement de leur pou-

9. Castells M., The Rise of the Network Society, Oxford, Blackwell, 1996, p. 3.

10. Shaw M., "Civil society and global politics: beyond a social movement approach", Millennium, vol.23, $\mathrm{n}^{\circ} 3$, 1994, pp. 644-665.

11. Held D., McGrew A., The Global Transformation Reader: An Introduction to the Globalization Debate, Cambridge, Polity Press, p. 72.

12. Deutscher Bundestag, 2001, p. 111. 
voir, le débat sur le « déficit de la démocratie » qui caractériserait ces organisations, pour la plupart non électives et peu transparentes quant à leur mode de fonctionnement (ce qui les rend peu sensibles aux protestations des citoyens) a également pris de l'importance. En réalité, les organisations intergouvernementales réunissent avant tout les gouvernements qui, bien que démocratiquement élus, le sont rarement pour les décisions qu'ils prendront au niveau international. A l'exception de l'Union européenne (où les pouvoirs du Parlement sont tout de même encore limités), ces choix ne prévoient pas d'assemblée représentative laissant un rôle à l'opposition. Dans les organisations comme la Banque mondiale ou le FMI, le poids décisionnel des différents pays dépend de leurs contributions économiques. Dans un grand nombre de cas, comme ceux des nombreux sommets contestés des nations les plus industrialisées (G7 et G8), les organisations intergouvernementales n'ont pas non plus de statut formel. Le manque de responsabilité démocratique de ces institutions a été considéré comme la cause d'une perte de pouvoir ultérieure des citoyens, gouvernés par des acteurs non élus et souvent peu visibles.

Seattle a permis de souligner que, même si la mondialisation réduit certains espaces pour l'action collective, de nouvelles opportunités s'offrent par ailleurs. Premièrement, la mondialisation culturelle, qui a pour conséquence une plus grande attention portée aux thèmes et aux problèmes des pays éloignés géographiquement, et l'accélération de la communication permettent la construction d'une sphère publique transnationale. Cette dernière est définie comme "un espace dans lequel les résidents des endroits distants (Etats ou localités) et les membres d'entités transnationales (organisations ou entreprises) élaborent des discours et des pratiques dont l'usage s'étend au-delà des barrières nationales $13 »$. Les nouvelles technologies, notamment Internet, ont réduit considérablement les coûts de communication, en permettant ainsi aux idées et aux projets de voyager rapidement à l'échelle mondiale. Que cela soit dans l'hémisphère nord ou sud, les processus culturels d'individualisation susmentionnés auraient élevé, du moins dans certains groupes, la conscience de leurs propres droits, ou la confiance en leur capacité à intervenir sur leur propre destin. Les valeurs démocratiques, généralement relayées par ces mêmes organisations gouvernementales internationales, auraient été prises au sérieux par les citoyens qui demandent de plus en plus à jouer un rôle.

La conviction la plus répandue dans les années 1990 selon laquelle la mobilité du capital, encouragée par les facteurs technologiques, détruit la capacité de la politique à gouverner les marchés, a été remise en question. En affirmant qu' "un autre monde est possible », les manifestations durant les

13. Guidry J.A., Kennedy M.D., Zald M.N. (eds.), Globalization and Social Movements: Culture, Power and the Transnational Public Sphere, Ann Arbor, University of Michigan Press, 2000, p. 7. 
sommets internationaux suggèrent la non-inéluctabilité du choix de la réduction des interventions politiques sur le marché et sur la pression fiscale qui entraîne le démantèlement de l'Etat social. Selon ces protestations, des mesures de libéralisation des échanges, en particulier des marchés financiers, ont été voulues et réalisées par des acteurs politiques spécifiques, soit à l'intérieur de différents Etats (notamment les plus puissants, les Etats-Unis d'Amérique), soit au niveau international, tout d'abord par les institutions financières internationales (Banque mondiale, FMI, OMC). C'est surtout dans la seconde moitié des années 1990 que les crises financières ont rendu visible l'insatisfaction produite par la doctrine du libre-échange. Ces crises ont eu lieu dans les pays de l'Asie de l'Est qu'on présente habituellement comme des exemples du succès des politiques néolibérales, puis en Argentine où les crédits du FMI étaient conditionnés par les privatisations et la dérégulation. Cependant, ces crises n'ont pas renforcé la conscience de la nécessité de créer des institutions mondiales pouvant gouverner une économie, manifestement incapable de s'autogouverner. Le déplacement des décisions au niveau supranational a favorisé à son tour le développement quantitatif des organisations non gouvernementales, comme nous l'avons déjà mentionné ${ }^{14}$. Il semble que ces organisations ont bénéficié par ailleurs, et particulièrement grâce au dynamisme des pays du Sud, d'une augmentation du nombre de leurs membres et de leurs ressources matérielles ${ }^{15}$.

Suite à Seattle, il est devenu évident que les différents phénomènes combinés de manière aléatoire, dans la définition de la mondialisation, produisent des conflits nouveaux, créant une structure complexe d'opportunités et de liens. Comme cela a été le cas à Prague, à Porto Alegre, à Göteborg et à Gênes, les divers acteurs impliqués dans ce mouvement de contestation se sont en outre " mis en réseau ", ce qui a donné naissance à une mobilisation commune. Malgré tout, une interrogation émerge, à la fois parmi les militants et parmi les commentateurs et les chercheurs, à travers la parole de trois intellectuels qui critiquent la mondialisation néolibérale 16 : la convergence des militants pour des protestations communes "donnera-t-elle vie à un mouvement mondial ou bien ces protestations ne sont-elles qu'un simple agrégat de mouvements séparés? "Bien que nous nous trouvions certainement face à de nouvelles vagues de mobilisations aussi bien au Sud qu'au Nord, peut-on véritablement parler d'un «mouvement social mondial »?

14. Deutscher Bundestag, 2001, p. 111.

15. Roth R., «NGOs und transnationale soziale Bewegungen: Akteure einer Weltzivilgesellschaft? ", in Brand U., Demirovic A., Goerg C., Hirsch J. (eds.), Nichtregierungsorganisationen in der Transformation des Staates, Münster, Westfälisches Dampfboot, 2001, pp. 43-63.

16. Brecher J., Costello T., Smith B., Globalization from Below. The Power of Solidarity, Cambridge, South End Press, p. 43. 


\section{La recherche sur les mouvements sociaux, la dimension transna- tionale et les pays du Sud}

Tout comme la protestation, la sociologie des mouvements sociaux a procédé par cycles, en se développant dans des phases de visibilité des mobilisations et, au contraire, en se consolidant dans les phases de latence de la mobilitation. A chaque vague, s'est jouée une adaptation des concepts et des théories concernant les caractéristiques des mouvements actifs à ce moment-là. Ainsi, dans la première phase de l'émergence des études sur les mouvements altermondialistes - influencés par les mouvements radicaux et les mouvements de protestation violente - l'approche dominante soulignait l'irrationalité de la masse ou, du moins, l'anomalie du comportement collectif par rapport aux formes normales de participation politique et sociale. Avec les mouvements apparus à la fin des années 1960, la sociologie des mouvements sociaux s'est enrichie en devenant, surtout aux Etats-Unis, un secteur bien visible et affirmé. L'image des mouvements a toutefois changé, comme l'approche dominante qui, désormais, souligne davantage la rationalité de la protestation et la capacité des organisations des mouvements sociaux en termes de mobilisation des ressources, en bénéficiant de l'ouverture des opportunités politiques. Avec le temps, les mouvements sociaux ont été étudiés comme un seul sujet et on a jugé leur forme d'action de plus en plus conventionnelle et leur structure organisationnelle, de plus en plus bureaucratique. Dans les années 1980, les chercheurs travaillant sur les mouvements sociaux se sont attachés à suivre les mouvements dans leurs différents parcours d'institutionnalisation, en se focalisant de nouveau sur les groupes d'intérêt collectifs, les entreprises actives dans le secteur tertiaire, l'opinion publique et les partis politiques émergents.

Le nouveau cycle de protestation, visible en 1997 avec la contestation du Millenium Round à Seattle, a été l'occasion (souvent bienvenue) de revenir aux études d'une "autre politique », mais aussi en partie un défi aux catégories héritées du passé. En effet, la recherche sur la «troisième vague» des mouvements confirme l'utilité de certains concepts et de certaines hypothèses élaborés dans le passé, mais aussi le besoin d'affiner l'analyse des processus restés marginaux, du moins dans l'approche «rationaliste » dominante : la construction de l'identité collective, la mobilisation des passions, l'élaboration de modèles alternatifs de démocratie 17.

Dans de nombreuses conférences et ouvrages dédiés à ce thème, les questions encore ouvertes concernent la nature du mouvement mondial - défini de façon variable comme «Noglobal», «New Global», "Globalizierungskritiker»,

17. Pour une présentation des différentes approches, voir par exemple: Cefai D., Pourquoi se mobilise-t-on? Les théories de l'action collective, Paris, La Découverte, 2007. 
«altermondialiste », ou comme un « mouvement pour une justice globale » 18 . La recherche sur les mouvements sociaux post-Seattle ${ }^{19}$ a confirmé l'importance croissante de la dimension globale de la protestation. Certes les éléments supranationaux (qui ont pour cible les réseaux et les schèmes de référence) étaient présents dans les mouvements du passé, mais la quantité et la qualité de la communication transnationale se sont accrues avec les nouvelles technologies et la mondialisation économique, politique et culturelle qu'on a presque ignorée. Les contestations de Seattle (et puis celles qui ont suivi à Prague, Göteborg et Gênes) ne représentent pas une rencontre occasionnelle d'une coalition ad hoc, mais elles s'inscrivent plutôt dans un parcours, entamé dans les années 1990. Il s'agit d'un parcours de croissance de ce que l'on a appelé la «société civile globale 20 ». Les chercheurs ayant travaillé sur le mouvement mondial ont souligné la progressive multiplication des organisations de mouvements sociaux transnationaux, une plus grande attention portée à la justice globale et à une mondialisation par le bas comme schème de référence central, et dans les protestations récentes, le cosmopolitisme relationnel des activistes, les campagnes de protestation supranationale, contre la cible internationale.

Les racines des mouvements ont conservé - qu'il s'agisse de leur position dans l'évolution historique des mouvements de gauche, ou de leur adaptation aux opportunités politico-institutionnelles particulières - leurs spécificités (couleur politique du gouvernement en place, diversité de la gauche institutionnelle). Aussi, même si l'on se focalise sur l'Europe ou sur l'hémisphère nord, ces différences sont fondamentales et sont prises en compte dans la recherche en sciences sociales ${ }^{21}$. L'attention portée au transnational n'a en effet pas exclu la dimension nationale : en dépit d'une fréquente stigmatisation (bien que peu documentée), d'une présumée tendance décontextualisante, la recherche sur les mouvements sociaux (sur les mouvements sociaux « mondiaux » également) est restée très ancrée dans les dimensions nationales. La dimension transnationale des mouvements récents est encore peu étudiée, étant donné l'intérêt récent porté aux mouvements sociaux (et aux autres acteurs non étatiques) dans les disciplines, à l'instar des relations internationales qui, auparavant, se concentraient sur la dimension supranationale et les racines de l'étude des mouvements sociaux dans les sciences sociales traditionnellement portées sur l'analyse du niveau local ou régional.

18. Della Porta D., The Global Justice Movement in Cross-National and Transnational Perspectives, Boulder, Paradigm, 2007.

19. Cette recherche est déjà assez riche, comme en témoigne, par exemple, la bibliographie publiée sur le site demos.it

20. Bandy J., Smith J. (eds.), Coalitions across Borders: Transnational Campaigns and the NeoLiberal Order, Lanham, Rowman \& Littlefield, 2005.

21. Della Porta D., The Global Justice Movement in Cross-National and Transnational Perspectives, op. cit. ; Sommier I., Fillieule O., Agrikoliansky E. (dirs.), Généalogie des mouvements altermondialistes en Europe, Paris, Karthala, 2008. 
Pour cette raison, et partant du pré-requis de l'existence d'un mouvement mondial, les différentes recherches empiriques enrichissent déjà notre connaissance sur un phénomène tout aussi récent qu'elle, le débat sur le degré d'innovation des mouvements récents est vif ${ }^{22}$, en partant de l'existence même d'un mouvement global. A l'instar de la définition de «mouvement social » utilisée dans l'analyse des mouvements nationaux ${ }^{23}$, on peut appeler mouvement social mondial un réseau transnational d'acteurs qui définissent leur cause comme mondiale et qui organisent des campagnes de protestation au niveau supranational. Ces trois éléments de la définition représentent les trois caractéristiques les plus discutées au sein de cette nouvelle vague de protestation.

Les mouvements sociaux sont des réseaux informels qui rassemblent en leur sein une pluralité d'acteurs. Une caractéristique distinctive des mouvements sociaux est leur capacité à « faire partie » d'une organisation spécifique sans y « appartenir ». Pour être mondial, un mouvement doit comprendre des organismes réticulaires actifs dans différents pays. En effet, le terme «transnational » a été de plus en plus souvent utilisé dans les sciences sociales pour indiquer l'importance croissante des acteurs non nationaux, des entreprises économiques multinationales aux ONG. La croissance de la « société civile mondiale » est représentée entre autres par des organisations transnationales de mouvements sociaux, que l'on compte désormais par milliers. C'est le reflet des différents phénomènes désignés par le terme plutôt flou de «mondialisation ». Mais bien que l'augmentation de l'importance numérique de ces organisations soit désormais avérée, les opinions des chercheurs varient concernant la capacité de ces groupes à se mettre en réseau et surtout à « se constituer en réseau » au-delà d'occasions ponctuelles. Une structure hautement flexible, avec des manifestations organisées en bonne partie via Internet, est en fait considérée par les uns comme une adaptation vitale à la société mondiale et, par d'autres, comme un signe d'une incapacité à construire des organisations pérennes. Le débat scientifique sur les formes que prennent et prendront ces réseaux est encore ouvert : coalitions occasionnelles ou réseaux de mouvements ? Phénomène mondial ou macro-régional (par exemple, au niveau européen) ? Majoritairement national (niveau principal d'intervention des mouvements historiques) ou local (dimension prévalente d'intervention desdits nouveaux mouvements) ? Et surtout, quelles conceptions de la démocratie accompagnent l'image d'un « mouvement des mouvements » ? Dans quelle mesure héritent-elles de la tradition participative des mouvements du passé et combien importent-elles de nouvelles conceptions de la démocratie délibérative, discutées dans les théories normatives et expérimentées dans la pratique ?

22. Della Porta D., Tarrow S. (eds.), Transnational Protest and Global Activism, Lenham, Rowman \& Littlefield, 2005.

23 . Della Porta D., Diani M., Social Movements: An Introduction, Oxford, Blackwell, 2006, chap. 1. 
Une deuxième caractéristique fondamentale d'un mouvement social est sa capacité à développer une identité collective, avec une interprétation de la réalité partagée. Outre les manifestations habituelles, les mouvements développent des systèmes de valeurs alternatifs ou, du moins, signalent l'émergence de nouveaux problèmes et de nouvelles solutions possibles. La différence de genre, la défense de l'environnement, la cohabitation de différentes cultures ont constitué des thèmes centraux, soutenus par des mouvements qui se sont développés au cours des dernières décennies. Pour être mondial, un mouvement doit développer un discours où l'on puisse reconnaître une identité commune (le «nous») et une cible de protestation («l'autre») au niveau mondial. Ici également, la recherche empirique a détecté l'augmentation des identifications cosmopolites, aussi bien chez les militants, que dans les documents émanant de leur organisation. Néanmoins, plusieurs questions restent ouvertes. L'appel aux valeurs mondiales est-il l'expression d'une réelle amplification du faisceau des intérêts des acteurs collectifs et individuels, ou bien une adaptation opportuniste (ou purement rhétorique) à une gouvernance territoriale à plusieurs niveaux ? Surtout, les thèmes de « la justice mondiale » ou de la «mondialisation par le bas » sontils véritablement capables de construire des identités, non seulement transnationales mais aussi trans-thématiques, liant d'une manière stable les identités collectives préexistantes d'écologistes, de féministes, de syndicalistes, etc. ? Et quel degré d'homogénéité de valeurs ${ }^{24}$ peut-on demander à un mouvement qui se définit comme pluriel et tolérant envers sa diversité interne ?

Enfin, les mouvements sociaux sont caractérisés par l'utilisation de formes de participation qui, bien qu'elles ne puissent plus désormais se définir comme non conventionnelles, ne sont certainement pas encore devenues routinières. En réalité, pour de nombreux chercheurs, la caractéristique des mouvements sociaux est d'agir à travers la protestation, c'est-à-dire une action dotée d'une charge innovatrice suffisante pour attirer l'attention des médias de masse et être ainsi (re)connue par l'opinion publique que l'on souhaite sensibiliser. En même temps, la protestation se présente comme une forme qui vient bouleverser et défier la capacité des gouvernants à s'assurer le consensus à travers les canaux normaux de la démocratie représentative. Bien que la plupart des actions de protestation soient encore orientées vers des objectifs nationaux, ou sub-nationaux, et si l'action visant les organisations de gouvernance internationale a souvent pris la forme discrète du lobbying, les actions de contestation supranationales ont été très médiatisées ces dernières années. Ces actions ont pris la forme de protestations (comme les mobilisations à Seattle contre l'OMC ou à Gênes contre le G8), mais également de campagnes supranationales (comme Jubileum 2000, puis Jubileum South pour l'annulation de la

24. Cet élément a été souligné par Rucht D., «Un movimento di movimenti ? Unità e diversità fra le organizzazioni per una giustizia globale », Rassegna Italiana di Sociologia, vol.46, n², 2005, pp. 275-306. 
dette extérieure des Etats les plus pauvres), ou des Journées d'action globale (comme les manifestations pacifistes du 15 février 2003). Ce phénomène d'amplification des formes de protestations supranationales est présent dans la recherche sociologique, laquelle s'est intéressée aux caractéristiques des protestations orientées vers des thématiques telles que les inégalités mondiales, la taxation des mouvements de capitaux, la consommation critique, les droits de citoyenneté au-delà des frontières, la réforme des organisations de gouvernance internationale. Restent toutefois ouvertes les interrogations sur la « viabilité » d'un niveau supranational de protestation, sûrement plus coûteux à maintenir par des réseaux informels d'acteurs pauvres en ressources.

La simple existence de ces débats témoigne de la difficulté d'adapter non seulement des catégories «nationales » à une dimension supranationale, mais aussi des catégories élaborées dans le Nord à des phénomènes qui impliquent également l'hémisphère sud (mais avec des tensions internes fréquentes). Si les interrogations susmentionnées sont présentes dans les recherches sur le mouvement pour une justice globale, d'autres interrogations peuvent s'y ajouter - comme le fait ce numéro de Cultures $\mathcal{E}$ Conflits - si la recherche se focalise sur le Sud. La réflexion sur les mouvements sociaux du Sud pourrait en effet aider à fournir un nouveau cadre, plus riche, au débat sur les mouvements récents et, en particulier, sur le mouvement reconnu en France sous le terme «altermondialiste».

En premier lieu, il s'agit d'observer combien les mobilisations sociales dans les pays en voie de développement qui se sont coordonnées dans le «mouvement mondial » sont différentes les unes des autres. Le choix d'organiser les rencontres du Forum social mondial à Porto Alegre, Mumbai, Karachi, Bamako et Nairobi "reflète la tentative explicite de la part des organisateurs du FSM de rendre les voix et les perspectives $d u$ Sud du monde plus centrales dans une discussion sur les politiques globales 25 », mais elle reflète également la force de ces mobilisations, qui ont porté les partis de gauche au pouvoir dans beaucoup de régions du Sud.

La mobilisation des populations indigènes a été très visible et culturellement significative. A partir des années 1980 surtout, du Canada au Mexique, en passant par l'Amérique centrale et méridionale, les populations indigènes ont protesté contre des projets spécifiques de construction de grandes infrastructures dans leur communauté ou, plus généralement, en faveur des formes d'autogouvernance non territoriales, souvent revendiquées à partir d'accords datant de l'époque de la première vague de colonisation. Au cours de campagnes transnationales, ces mobilisations ont été portées à la reconnaissance internationale, notamment à travers la Charte des droits des populations indigènes de l'ONU. Les accords de libéralisation économique ont cependant été accusés de violer les droits de ces populations, considérés comme des sources de protec-

25 . Smith J., et al., op. cit., p. xi. 
tionnisme. Aux origines du mouvement zapatiste au Mexique, il y a la défense du ejido, c'est-à-dire des terres communes de tradition maya, reconnues comme telles dans l'article 27 de la Constitution mexicaine, abrogé après les accords de libéralisation économique entre le Mexique, le Canada et les EtatsUnis, l'ALENA 26. C'est en réalité le jour même de l'entrée en vigueur de l'ALENA, le 1er janvier 1994, que l'Ejército Zapatista de Liberación Nacional (EZLN) a initié sa protestation au Chiapas. Pour la défense des populations indigènes, désignées comme les principales victimes du néolibéralisme, la mobilisation du Chiapas revendique le droit à une vie en harmonie avec la nature, contre l'exploitation sans règles et le développement sauvage imposé par les multinationales et les organisations financières internationales.

Les revendications des populations indigènes se sont ensuite souvent entrecroisées, surtout au Sud, avec celles des paysans, par exemple dans l'opposition aux «Brevets sur le vivant», prévus par l'Accord sur les droits de propriété intellectuelle liée au commerce (ADPIC), en vigueur depuis 1995. Au cours des années 1990 en Inde, les paysans ont protesté contre la multinationale Monsanto, qui avait inventé et mis sur le marché des semences transgéniques stériles, et contre les brevets accordés par les Etats-Unis, aussi bien concernant l'utilisation d'une plante, déjà utilisée traditionnellement pour ses diverses vertus (le Neem), que sur l'espèce de riz Basmati, également traditionnellement cultivée dans ce pays. Outre le refus des brevets sur les êtres vivants et les plantes, il existe d'autres revendications présentes dans la plateforme de Via Campesina, organisation «parapluie » de groupes de paysans actifs dans plusieurs pays. Il s'agit de la «souveraineté alimentaire » (par extension, le droit de chaque population à définir les politiques agricoles et alimentaires durables qui garantissent le droit à l'alimentation et l'accès aux ressources de base); la protection de la biodiversité, face à un libre marché accusé de conduire les pays les plus pauvres vers la monoculture ; une réforme agraire qui distribue la terre aux paysans et réintroduit les terres communes ; le refus des produits génétiquement modifiés ; une réelle ouverture du commerce pour les produits du Sud. Fondée en 1993, la Via Campesina, qui avait impliqué 69 organisations de 37 pays lors de sa deuxième conférence en 1996 à Mexico, concerne aujourd'hui des organisations comptant 50 millions d'inscrits dans 50 pays. Comme les populations indigènes au Canada, les paysans et les populations indigènes d'Inde ou de Thailande ont protesté contre les projets de construction de grandes digues et les relocalisations forcées des habitants des zones concernées qui s’en suivaient. Si, parmi les leaders les plus visibles du mouvement on retrouve le paysan français José Bové, avec sa Confédération paysanne, au deuxième Forum social mondial de Porto Alegre étaient présentes, aux côtés des Sem Terra (Sans-terre) brésiliens, des organisations d'ouvriers agricoles d'Argentine, du Belize, du Chili, du Costa

26. De Angelis M., “Globalization, New Internationalism and the Zapatistas”, Class and Capital, n70, pp. 9-35. 
Rica, de Cuba, d'Equateur, des Philippines, du Guatemala, du Honduras, du Mexique, du Nicaragua, du Panama, du Paraguay, du Pérou, d'Inde, d'Indonésie, de Madagascar et de Colombie 27.

Dans de nombreux pays du Sud, des manifestations, parfois violentes, ont remis en cause les mesures d'austérité imposées par des "ajustements structurels ", mis en œuvre par les gouvernements nationaux sous l'égide du FMI et de la Banque mondiale ${ }^{28}$. Bien que, traditionnellement, les institutions de Bretton Woods aient favorisé, dans différents pays du Sud, des politiques d'expansion des dépenses publiques, elles ont toutefois été largement accusées d'avoir trahi par la suite leur mandat d'origine. En effet, surtout à partir des années 1980, au travers des Programmatic Structural Adjustment Credits, ces institutions ont prôné des politiques déflationnistes et libérales avec, suivant le cas, des réductions de la dépense publique et donc des services, l'élimination des subventions et $\mathrm{du}$ contrôle des prix de certains produits, la réduction des impôts, notamment sur les capitaux, la privatisation des services publiques, l'abolition des terres communes, les dérèglementations du marché du travail, la dévaluation de la monnaie locale (avec la perte de pouvoir d'achat des salariés). Parmi les conséquences de ces politiques, souvent dénoncées par les ONG, on compte l'amplification de la pauvreté, de l'analphabétisme, des famines et des épidémies, ainsi que de la violence interethnique ${ }^{29}$. Notamment après la crise au Mexique, chez les «tigres asiatiques » et en Argentine, des grèves et des protestations ont été organisées par des groupes communautaires ainsi que par des syndicats. Entre janvier et septembre 2000 seulement, on a compté 48 manifestations majeures contre les privatisations et les mesures d'austérité dans 13 pays latino-américains ${ }^{30}$. En Argentine, où le régime des colonels avait reçu des crédits internationaux directement déposés sur des comptes bancaires étrangers et utilisés pour leur enrichissement personnel, les politiques économiques de privatisation et dérèglementation sont accusées d'avoir conduit la moitié de la population sous le seuil de pauvreté ${ }^{31}$. Ces politiques, ont entraîné la mobilisation des Piqueteros, des chômeurs avec des expériences professionnelles et syndicales, dans une «étrange alliance » avec les classes moyennes appauvries, protagonistes des protestations des cacerolazos (casseroles utilisées comme des tambours). Surtout, des assemblées de barrio se sont constituées dans les quartiers des grandes cités, (en 2001, 272 barrios ont été recensés, dont $40 \%$ à Buenos Aires), qui organisent entre autres des coopératives d'achat et d'échange en nature à travers la Red del Trueque Solidario

27. Jampaglia C., Bandinelli T. (eds.), Porto Alegra. Il Forum Sociale Mondiale, Milano, Feltrinelli, 2002, p. 43.

28. Walton J., Seddon D., Free Markets and Food Riots. The Politics of Global Adjustment, Oxford, Blackwell, 1994.

29. Mies M., Globalizierung von unten. Der Kampf gegen die Herrschaft der Konzerne, Hamburg, Europäische Verlagsanstalt, 2002, pp. 75-76.

30. Lichbach M.I., Amed P., Global Order and Local Resistance: The Neoliberal Institutional Trilemma and the Battle of Seattle, University of California, Riverside, 2001, p. 52 et suivantes.

31. Mies M., op. cit., p. 75. 
(littéralement «Réseau de troc solidaire »). Les syndicats, notamment sudcoréens, et la Central Unica dos Trabalhadores du Brésil sont également particulièrement présents avec leurs propres représentants aux manifestations contre la mondialisation néolibérale ${ }^{32}$.

Last but not least, la même mondialisation culturelle, couplée à l'interventionnisme croissant des ONG et à la promotion de processus de démocratisation dans de nombreux pays, a contribué, dans le Sud, au développement d'une sensibilité pour des thèmes habituellement considérés comme postmatérialistes ${ }^{33}$. A titre d'exemple, des organisations de femmes se sont développées et se sont rencontrées, soit au cours de conférences financées par l'ONU, soit lors de rencontres transnationales autonomes. Parmi ces organisations, on compte le mouvement des femmes indiennes Chipko, le RAWA (Revolutionary Association of Women in Afghanistan) et le Green Belt Movement, qui mobilise 80000 femmes au Kenya. Lors des mobilisations contre la construction de barrages, nées en particulier sur la base de la défense du travail des femmes, les thématiques écologiques sont devenues de plus en plus importantes. La campagne de protestation contre la construction d'une grande digue au sud du Brésil, entamée en 1979, en tant que revendication des droits au logement et au travail pour ceux qui auraient été chassés de leur terre, a également inclus, en 1988, un discours sur la défense de l'environnement, après que les populations locales, protagonistes des premières actions collectives, aient rencontré des ONG, conduisant à une " contamination » réciproque ${ }^{34}$. De même, au Chiapas, des expériences d'autogestion se sont développées au moyen d'une révision des formes traditionnelles de gestion communautaire des biens communs et de l'influence des expériences de plusieurs mouvements dans différentes régions du monde 35 .

Lors des manifestations des populations indigènes et paysannes, des femmes et des environnementalistes, les institutions financières internationales se voient accusées d'avoir reproduit et accentué les inégalités, la pauvreté, la destruction de l'environnement.

En dépit de l'importance du mouvement altermondialiste au Sud (de l'Amérique latine à l'Inde) et de l'intensification du flux d'idées et d'expériences provenant des mouvements d'autres continents (du preguntar caminando du zapatisme au Mexique au bilan participatif de Porto Alegre), la

32 . Josselin D., "Trade unions in a global age”, in Josselin D., Wallace W. (eds.), Non-state Actors in World Politics, Basingstoke, Palgrave, 2001, pp. 169-186.

33. Morris-Suzuki T., "For and against NGOs", New Left Review, n², 2000, pp. 63-84.

34 . Rothman F., Oliver D.E., Oliver P.E., "From local to global: the anti-dam movement in Southern Brasil,1979-1992", in Imig D., Tarrow S. (eds.), Contentious Europeans. Protest and Politics in an Emerging Polity, Lanham, Rowman \& Littlefield, 2002, p. 128.

35 . Benenati S., Storia del Chiapas. Gli zapatisti e la rete sociale globale, Milano, Mondadori, 2002, p. 37. 
recherche sociologique sur le mouvement pour une justice globale est restée principalement focalisée sur le Nord ${ }^{36}$. L'enracinement de la recherche sur les mouvements sociaux - ou du moins sur leurs courants les plus visibles au Nord, et la concentration de l'attention des spécialistes venant d'autres aires géographiques sur des dimensions davantage institutionnelles de la politique, explique en partie pourquoi la recherche sur le mouvement pour une justice globale (ou altermondialiste, ou antilibérale...) au Sud est certainement encore marginale. Les chercheurs des mouvements sociaux et les chercheurs du Sud ont eu encore peu d'occasions de rencontre et de « contamination» réciproque. Etant donné que le concept de «mouvement social» (comme la plupart des concepts de science et de sociologie politiques) a été forgé dans des contextes démocratiques, son potentiel d'utilisation semble limité dans des régimes autoritaires ${ }^{37}$ ou dans des contextes de transition vers la démocratie. Mais, parallèlement à cela les approches dominantes sur les mouvements sociaux du Nord ne sont pas très utilisées pour étudier ceux du Sud. A titre d'exemple, en Amérique latine on a parlé de "mouvements sociaux » dans le cadre de la théorie de la dépendance et de l'approche marxiste, alors que celles-ci restent peu utilisées par les chercheurs travaillant sur les mouvements sociaux de l'hémisphère nord. Ou encore, les hypothèses construites sur les mouvements sociaux post-matérialistes et les «nouvelles classes moyennes » du Nord s'adaptent peu à l'étude des mouvements sociaux du Sud. Dans les pays du Sud, non seulement la dimension de classe émerge avec puissance, mais elle s'entrecroise avec des questions nationales, des discours sur la souveraineté, l'identité religieuse, etc. L'unité des catégories et des hypothèses formulées par la sociologie des mouvements sociaux doit être, par conséquent, vérifiée non seulement historiquement, vis-à-vis d'un nouveau cycle de protestation, mais aussi géographiquement.

Les études de cas sur la société civile globale et les mouvements dans l'hémisphère sud semblent confirmer une grande partie des observations faites dans d'autres aires géographiques. Parmi celles-là, les mille visages des campagnes transnationales qui impliquent les acteurs les plus divers, la radicalisation des ONG et la re-mobilisation des syndicats sur les thématiques sociales, l'importance (non seulement rhétorique) de stratégies globales, la présence de stratégies multiples (de l'éducation populaire aux manifestations de rue), un trans-nationalisme hétérogène. A titre d'exemple, le travail de Gülçin Lelandais ${ }^{38}$ sur le mouvement altermondialiste en Turquie souligne

36. Della Porta D., Tarrow S., op. cit.

37. Boudreau V., Resisting Dictatorship. Repression and Protest in Southeast Asia, Cambridge, Cambridge University Press, 2004 ; Bennani-Chaibi M., Fillieule O., « Exit, voice, loyalty and bien d'autres choses encore ", Résistances et protestations dans les sociétés musulmanes, Paris, Presses de Sciences Po, 2003, pp. 43-126.

38 . Erdi Lelandais G., "Cosmopolites enracinés" : La transnationalité et la constrution identitaire du mouvement altermondialiste: l'exemple de la Turquie, thèse de doctorat, Paris, EHESS, 2007 ; et voir son article dans ce numéro. 
la présence, également dans ce pays, d'une structure organisatrice réticulaire, qui implique des organisations plus ou moins formalisées ; l'invention de nouveaux répertoires d'action, avec un accent particulier placé sur la créativité et le sens symbolique de l'action collective ; la création d'arènes de délibération qui, comme les forums sociaux, fournissent une occasion de rencontre aux activistes d'organisations d'idéologies et d'intérêts différents ou bien, comme souvent dans le passé, dans une compétition réciproque forte, accentuée par un régime répressif ; l'importante attention accordée aux thèmes sociaux, avec tout de même un schéma de référence innovant par rapport au discours plus traditionnel du mouvement ouvrier. Là encore, cette recherche confirme l'importance de la dimension traditionnelle de ces mobilisations collectives, en les plaçant dans un contexte géopolitique plus vaste. Ces similitudes (en bonne partie inattendues) devraient aider à dépasser les stéréotypes sur une sorte de retard culturel et stratégique des mobilisations dans l'hémisphère sud (stéréotypes reproduits, par exemple, par la focalisation de l'attention sur la dimension violente des mobilisations islamiques).

En même temps, les recherches ici présentées semblent toutefois faire appel à une réflexion sur quelques-uns des concepts les plus significatifs des études sur les mouvements sociaux. Premièrement, les bases sociales de la protestation paraissent bien plus variées dans les pays du Sud qu'en Europe. Le retour de la question sociale - proclamé également en Europe - revêt une importance encore plus forte au Sud, ce qui explique également la persistance d'une plus grande attention portée à la dimension de classe que dans l'hémisphère nord, où la recherche sur les nouveaux mouvements sociaux aurait plutôt déplacé l'attention accordée aux nouvelles classes moyennes. L'article sur le FSM de Nairobi coécrit par Marie-Emmanuelle Pommerolle et Johanna Siméant dans ce numéro nous démontre que, malgré leur diversité, toutes les voix africaines accordent une forte attention à la lutte contre la pauvreté.

En ce qui concerne le rôle de la structure des opportunités politiques, ce qui apparaît comme central - et cependant sous-évalué dans les études sur les pays du Nord - c'est la réflexion sur les caractéristiques des régimes autoritaires, ainsi que sur les parcours de démocratisation. La question de la démocratie émerge également de façon différenciée, avec la stigmatisation du rôle du Nord dans le soutien aux régimes autoritaires du Sud, mais aussi avec une redéfinition critique d'un concept de démocratie développé et imposé par le Nord.

Même s'il est vrai que la dimension nationale des opportunités politiques demeure décisive, ces études démontrent que les ONG internationales ont dans le Sud une présence et un rôle plus significatifs qu'en Europe, en tant que fournisseurs de ressources logistiques, mais aussi de modèles culturels contestés. Ainsi, la mobilisation des ressources - autre concept central des études sur les mouvements sociaux - se présente ici comme directement liée à la dynami- 
sation de groupes-réseaux complexes, avec des stratégies de boomerang ${ }^{39}$ et des alliances souvent difficiles avec les ONG internationales ${ }^{40}$.

De même, le phénomène de la mondialisation dans l'hémisphère Sud présente certaines spécificités en raison de son étroite liaison avec une pluralité de conflits. Par exemple, l'altermondialisme en Turquie doit s'orienter vers des axes de conflit différents : à la traditionnelle dimension de classe s'ajoutent des conflits entre le centre et la périphérie (avec l'importance de la question kurde), des conflits entre l'Etat et l'Eglise (particulièrement avec les tensions entre la laïcité et les organisations islamiques), des questions de souveraineté nationale, de démocratisation et de droits de l'Homme ${ }^{41}$. En particulier, dans le contexte africain, la dimension religieuse est très présente : dans la tradition d'intervention humanitaire et de soutien au développement par des organisations chrétiennes 42 mais également dans l'importance, pas toujours explicitées, de l'identité religieuse islamique. Dans l'élaboration de la protestation, un élément semble central (et certainement négligé dans les recherches sur les mouvements de l'hémisphère nord) : la dimension anti-impérialiste / anticoloniale de la protestation, étroitement liée à la définition de l'identité nationale, voire au nationalisme.

Enfin, les caractéristiques spécifiques des élites de ces mouvements (les cosmopolites enracinés dont parlent de nombreux articles ici réunis) revendiquent une attention particulière aux contextes sociaux et politiques caractérisés par une distribution des ressources de mobilisation bien plus inégales que celles que nous sommes habitués à considérer dans l'hémisphère nord.

39. Keck M., Sikkin K., Activists without Borders, Ithaca, Cornell University Press, 1998.

40. Voir dans ce numéro l'article d'Eric Cheynis et l'article de Marie-Emanuelle Pommerolle et Johanna Siméant.

41. Erdi Lelandais G., op. cit.

42 . Voir les articles d'E. Cheynis et de M.-E. Pommerolle et J. Siméant dans ce numéro. 